\title{
Self-Calibrating 3D-Ultrasound-Based Bone Registration for Minimally Invasive Orthopedic Surgery
}

\author{
Dean C. Barratt*, Member, IEEE, Graeme P. Penney, Carolyn S. K. Chan, Mike Slomczykowski, Timothy J. Carter, \\ Philip J. Edwards, and David J. Hawkes
}

\begin{abstract}
Intraoperative freehand three-dimensional (3-D) ultrasound (3D-US) has been proposed as a noninvasive method for registering bones to a preoperative computed tomography image or computer-generated bone model during computer-aided orthopedic surgery (CAOS). In this technique, an US probe is tracked by a 3-D position sensor and acts as a percutaneous device for localizing the bone surface. However, variations in the acoustic properties of soft tissue, such as the average speed of sound, can introduce significant errors in the bone depth estimated from US images, which limits registration accuracy. We describe a new self-calibrating approach to US-based bone registration that addresses this problem, and demonstrate its application within a standard registration scheme. Using realistic US image data acquired from 6 femurs and 3 pelves of intact human cadavers, and accurate Gold Standard registration transformations calculated using bone-implanted fiducial markers, we show that self-calibrating registration is significantly more accurate than a standard method, yielding an average root mean squared target registration error of $1.6 \mathrm{~mm}$. We conclude that self-calibrating registration results in significant improvements in registration accuracy for CAOS applications over conventional approaches where calibration parameters of the 3D-US system remain fixed to values determined using a preoperative phantom-based calibration.
\end{abstract}

Index Terms-Calibration, computer-aided surgery, orthopedics, registration, ultrasound.

\section{INTRODUCTION}

$\mathbf{T}$ ECHNOLOGICAL advances over the last decade have made systems for computer-aided orthopedic surgery (CAOS) increasingly available, with several commercial and

Manuscript received August 27, 2005; revised November 18, 2005. This work was supported by the Engineering and Physical Sciences Research Council (EPSRC) under Medlink Grant GR/R03525/01 (industrial partners: Depuy International-a Johnson \& Johnson Company, Brainlab AG, and Philips Medical Systems). Asterisk indicates corresponding author.

*D. C. Barratt was with the Department of Imaging Sciences, Guy's Hospital, GKT School of Medicine, King's College London, London SE1 9RT, U.K. He is now with the Centre for Medical Image Computing, Room 2.20, Malet Place Engineering Building, University College London, London WC1E 6BT, U.K. (e-mail: d.barratt@ucl.ac.uk).

G. P. Penney, C. Chan, T. J. Carter, and D. J. Hawkes were with the Department of Imaging Sciences, Guy's Hospital, GKT School of Medicine, King's College London, London SE1 9RT, UK. They are now with the Centre for Medical Image Computing, University College London, London WC1E 6BT, U.K.

M. Slomczykowski is with Depuy International-a Johnson \& Johnson Company, Leeds LS11 0EA, U.K.

P. J. Edwards was with the Department of Imaging Sciences, Guy's Hospital, King's College London, London SE1 9RTU.K. He is now with the Department of Biosurgery and Surgical Technology, St. Mary's Hospital, Imperial College London, London W2 1NY, U.K.

Digital Object Identifier 10.1109/TMI.2005.862736 research systems now well-established. Such systems assist the surgeon by providing enhanced methods for preoperative surgical planning and simulation, intraoperative navigation, and precise execution of robotic surgery [1]-[5]. The principal motivations for CAOS are increased surgical confidence and accuracy - for example, for optimal placement of prosthetic implants-and minimal invasiveness. These benefits are likely to lead to reduced perioperative and postoperative complications, improved postoperative function, shorter recovery time, and a reduction in the likelihood of revision surgery being required [5], [6].

To date, CAOS has been applied to a number of orthopedic procedures including total hip replacement (THR) [1], [2], [6]-[10], total knee replacement [1], pedicel screw insertion [11], [12], periactetabular osteotomy [13], tibial osteotomy [14], distal radius osteotomy [15], anterior cruciate ligament reconstruction [16], [17], osteoid osteoma excision [18], bone tumor resection [19], [20], and fracture surgery [21]-[23]. Further clinical applications will doubtless emerge as the demand for common procedures, such as THR, continues to increase [24], [25]. This increase in demand, combined with raised expectations concerning postoperative quality of life among the patient population, provide strong motivations for realizing the potential benefits of CAOS.

One area that has been the focus of recent attention is the development of more accurate and less invasive methods for registering a 3-D representation of bone anatomy to the patient in the operating theater. Registration is a critical step for any CAOS system that supports surgical navigation, and is widely considered to be the most significant factor which currently limits intraoperative navigation accuracy [1], [26].

In conventional computed tomography (CT)-guided CAOS systems, registration is achieved using either point- or surfacebased registration [1], [4], [27]-[33]. In the former, points on anatomical landmarks, bone-implanted fiducial markers, or an external fixation device, are identified in the operating theater using a tracked digitizer and registered to corresponding points in a preoperative CT image. In the surface-based approach, the surgically exposed bone surface is digitized. The resulting surface (commonly represented by a set of points) is then matched to the corresponding region on a bone surface model extracted from the CT scan. Recently, so-called "CT-free" systems have emerged in which a patient-specific bone model is generated and registered simultaneously by deforming a generic model to fit the digitized bone surface [34], [35]. Such systems have the ad- 
vantage that the cost and radiation dose associated with a preoperative CT scan are eliminated.

Although the accuracy of standard registration methods used for CAOS is generally accepted as sufficient for surgical navigation, with published errors between 0.5 to $5 \mathrm{~mm}$ [12], [26], [27], [30], [32], [36], these methods are very often highly invasive. There is, therefore, an associated risk of perioperative and postoperative complications, such as blood loss, infection, etc. due to soft tissue trauma [6], [37], [38].

To overcome this problem, ultrasound (US) has been proposed as a noninvasive alternative for localizing bone surfaces [30], [39]. In this approach, an US transducer is tracked in 3-D by attaching a position sensor. This technique, which is generally known as freehand 3D-US imaging, is a well-established method of generating 3-D diagnostic images. However, in the context of CAOS, the US image can be thought of intuitively as a percutaneous digitizer, which replaces a physical digitizer, and US-derived surface points used as input data for a standard surface-based registration scheme.

3D-US-based registration is attractive because it is safe, noninvasive and relatively inexpensive. Consequently, several research groups have investigated the technique for registering the skull [40]-[42], spine [11], [12], [30], [43]-[46], pelvis [47]-[50], femur [39], [51]-[53], and tibia [51]. However, very little attention has been given to the problem of errors in the measured bone location that arise as a result of variations in the acoustic properties of soft-tissue. In particular, deviation from the assumed value for the average speed of sound is recognized as a significant source of localization error [34], [54]-[56]. Conventionally, the speed of sound is assumed to be constant in diagnostic US systems with a nominal value of $1540 \mathrm{~m} / \mathrm{s}$. However, the actual speed of sound in human soft tissue ranges from approximately $1400 \mathrm{~m} / \mathrm{s}$ (in fat) to $1650 \mathrm{~m} / \mathrm{s}$ (in muscle) [57]. Based on a simplistic model of US propagation, which ignores additional effects such as refraction, such speed of sound variations can give rise to systematic errors in locating the depth of the bone surface of up to 5\%. Such errors can have a significant and unpredictable effect on registration accuracy, and may be particularly problematic in the obese patient where they can translate into an absolute bone localization error on the order of several millimeters.

Parameters such as the average speed of sound are very difficult to estimate accurately in vivo using direct methods [54], [56]-[60]. We have developed a new approach in which in vivo US data acquired for the purpose of noninvasive bone registration are simultaneously used to update the freehand 3D-US system calibration parameters. Underlying our approach is the notion that localization errors arising in vivo may be partially modeled as an error in these calibration parameters. The standard method for calibrating freehand 3D-US systems is to scan a special-purpose phantom containing one or more objects of known geometry [61], [62]. However, the ultrasonic properties, such as the speed of sound, within a phantom are generally well-characterized and may differ appreciably from those found in vivo, potentially leading to the phantom-based calibration parameters becoming sub-optimal in the sense that more accurate values may exist for in vivo imaging. In our approach, this is taken into account by intraoperatively updating calibration parameter values, determined using one-off preoperative phantom calibration, at the same time a registering US-derived surface points to a bone surface model. This is achieved by including them as free parameters in the registration optimization. In this method, the bone is effectively used as an in vivo calibration object, and the registration algorithm becomes "self-calibrating." This approach has the advantage of improving the registration accuracy by updating the calibration parameters to better reflect their optimal values based on in vivo image data.

In this paper, we investigate the accuracy and robustness of a self-calibrating, surface-based registration algorithm using data obtained from intact human cadavers, and compare its accuracy with a conventional algorithm where the calibration parameters are fixed.

\section{METHODS}

\section{A. Cadaver Preparation}

Three complete female cadavers with intact tissue, which differed in size and fat composition, were used in this study. These were provided by the Institute of Anatomy at Ludwig-Maximilian University (LMU) in Munich where all experiments involving the cadavers were carried out. For ethical reasons, our requirement for an accurate Gold Standard registration based on bone-implanted fiducial markers precluded the use of patients. Consequently, cadavers were chosen as the best substitute. The cadavers were stored at room temperature and had been preserved using a technique developed by Thiel [63]. This preservation method allowed realistic US images to be acquired and had the advantage that muscle flexibility was maintained, which enabled cadavers to be manipulated in the same manner as an anaesthetized patient undergoing surgery.

With the cadavers lying in the supine position, titanium bone screws (Part no. 80-09051, Stryker-Leibinger UK Ltd., Newbury, Berkshire, UK) with a $2 \mathrm{~mm}$ diameter threaded tip were implanted into both femurs and each side of the pelvis. Four bone screws were inserted into each femur, one near to the greater trochanter with the other three distributed along the lateral and anterior sides of the femoral shaft. For each hemi-pelvis, one screw was inserted into the pubis bone, and four distributed along the anterior iliac crest. In all cases, bone screws were implanted at surgically accessible locations with care taken to limit the amount of damage to the surrounding tissue in order to maximize the number of regions in which useful US images could be obtained. The centers of the bone-implanted fiducial markers were defined manually in the CT images using software developed by our research group.

\section{B. CT Imaging}

Once bone screws had been implanted, custom-made fiducial markers were attached to the end of each screw. Markers were filled with a radio-opaque contrast agent (Urografin 370, Scherring Healthcare Ltd., West Sussex, U.K.) so that they could be easily identified in a CT image. The cadavers were then transported to the Radiology Department where a high-resolution spiral CT scan of each cadaver was performed using a Siemens 


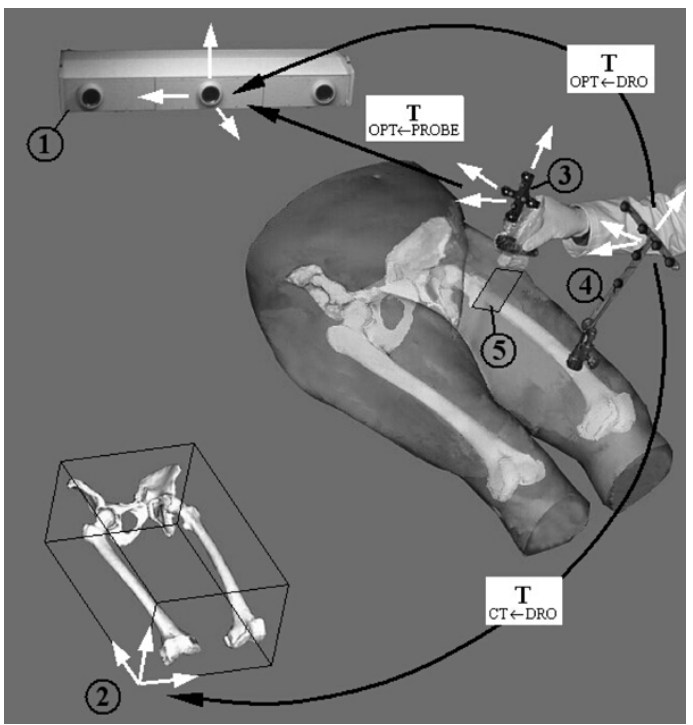

Fig. 1. Schematic of the experimental setup used for the validation study described in this paper: 1) Camera head of the Optotrak optical localizer (OPT); 2) CT image (CT); 3) Tracked object attached to the US probe (PROBE); 4) Dynamic reference object (DRO); 5) Ultrasound image (US). The labels used to denote the 3-D coordinate systems, shown as white arrows, are given parentheses. The black arrows indicate the rigid-body transformations between coordinates systems. The notation $\underset{\mathrm{B} \leftarrow \mathrm{A}}{\mathrm{T}}$ is used to denote a rigid-body transformation from 3-D coordinate system $\mathrm{B} \leftarrow$ to $\mathrm{B}$.

SOMATOM Plus 5 CT scanner (Siemens Medical Solutions, Erlangen, Germany). The field-of-view was such that the pelvis and both femurs were included in each scan. CT images were reconstructed with voxel dimensions between 0.7 and $0.8 \mathrm{~mm}$ in the transverse plane and a slice thickness of $2 \mathrm{~mm}$. Bone surfaces were segmented in the CT images by using the semi-automatic tools provided by the software package ANALYZE v. 6.0 (Mayo Foundation, Rochester, MN).

\section{Gold Standard Registration}

Following CT scanning, the cadavers were returned to the anatomy laboratory and the contrast-filled fiducial markers replaced with another type of fiducial marker containing a $3 \mathrm{~mm}$ diameter hemi-spherical divot. These markers were machined so that the center of the divot coincided with the centroid of the contrast-filled chamber of the imaging markers. This enabled the 3-D position of the centroid of the chamber to be located accurately in physical space by inserting a $3 \mathrm{~mm}$ ball-tip digitizer, tracked by a high-accuracy optical localizer (Optotrak 3020, Northern Digital Inc., Ontario Canada), into the divot and measuring the position of the center of the ball-tip.

The 3-D position of each fiducial marker was recorded relative to a tracked dynamic reference object (DRO) with 6 infrared light emitting diodes (LEDs) shown in Figs. 1 and 2. The DRO was rigidly fixed to a steel post inserted into the bone using standard surgical adaptors. This object was also tracked by the optical localizer and defined the reference coordinate system for physical space. The use of a DRO is standard practice in conventional CAOS as it enables the patient to be repositioned during surgery without the need for re-registration. The Gold

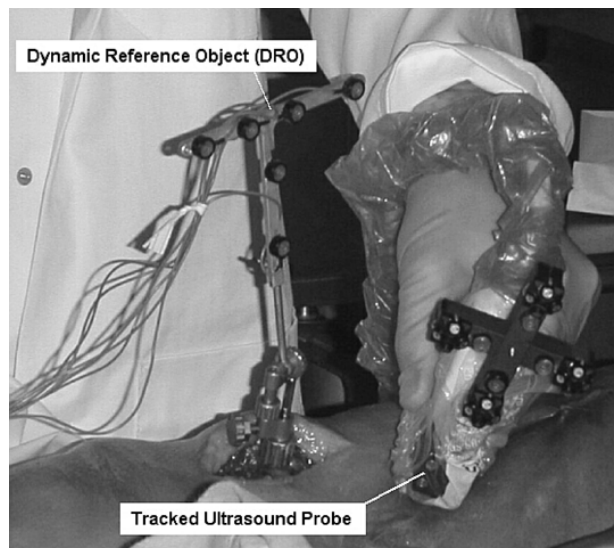

Fig. 2. US scanning of a cadaveric femur. The handheld US probe is shown on the right with a tracked object (position sensor) rigidly attached (see also Fig. 3). The DRO implanted into the femur is shown of the left.

Standard DRO-to-CT registration transformations were computed for each bone by registering together corresponding fiducial pointsets. In addition, the locations of the centers of rotation of the left and right hip joints were estimated by tracking the DRO attached to the distal femur while the leg was moved slowly through approximately $45^{\circ}$ of flexion and $30^{\circ}$ of abduction and adduction. Two-hundred position measurements were recorded, and the center of rotation was found by calculating the location of the invariant point relative to the DRO coordinate system. In this experiment, the center of rotation was assumed to remain unchanged whilst the leg was moved. Therefore, the pelvis was not simultaneously tracked using a second DRO. This assumption is justified by the low RMS residual error $(<1 \mathrm{~mm})$ calculated for the center of rotation of the hip joint.

\section{US Imaging}

Fig. 1 shows a schematic of the experimental set-up used for the cadaver study, with the rigid-body transformations that relate the 3-D coordinate systems of the various elements indicated. A diagnostic US scanner (Philips-ATL HDI-5000, Philips Medical Systems, Bothell, WA) with a high-frequency linear-array scan-probe (L12-5 probe; 5-12 MHz broadband transducer) was used to obtain US images for this study. The scan-probe was tracked using a custom-made attachment with an array of 20 LEDs, as shown in Figs. 2 and 3. The optical localizer measured the 3-D position of each LED, which were used to calculate the position and orientation of the attachment relative to the DRO. Custom-designed acquisition software, written in $\mathrm{C}++$ using Visual Studio 6 (Microsoft Corp., USA), was used to synchronize US image capture with tracking measurements. US images were captured using an analogue-to-digital converter (Canopus ADVC-100, Canopus UK, Berkshire, U.K.) connected between the composite video output of the US scanner and the Firewire (IEEE 1394) port of a laptop PC. The rigid-body calibration transformation for the 3D-US system, indicated by $\underset{\mathrm{PROBE} \leftarrow \mathrm{US}}{\mathbf{T}}$ in Fig. 3, was determined using the reference calibration method described in the next section.

During US scanning, the scan-probe was covered with a plastic sheath containing a small quantity of US gel to maintain 


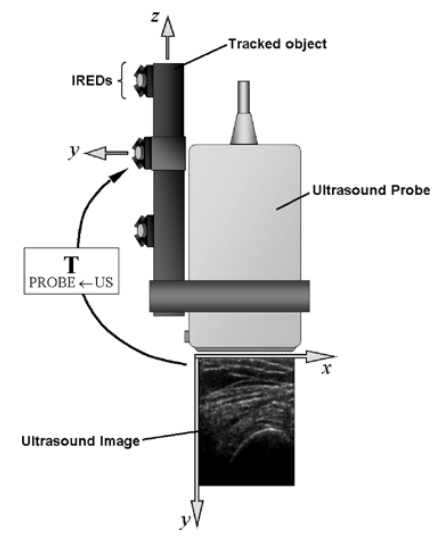

Fig. 3. The B-mode US probe with tracked attachment used for freehand 3D-US imaging in this study. The tracked object comprises 20 infrared LEDs and is rigidly fixed to the US probe. The gray arrows represent the axes of the 3-D coordinate systems of the tracked object and the US image, denoted in the text as "PROBE" and "US," respectively. (Note: the $x$-axis of PROBE and $z$-axis of US are not shown and are directed into the page). The black arrow represents the rigid-body transformation from US to PROBE coordinates.

acoustic coupling. In order to minimize motion artefacts and ensure that captured images were closely synchronized with the tracking data, the scan-probe was swept across the skin surface slowly $(<5 \mathrm{~mm} / \mathrm{s})$. For each cadaver, tracked US images of the surface of the femurs and pelvis were acquired from many different views: In the case of the femur, images were obtained of the anterior and (by lifting the leg) the posterior femoral shaft, the greater trochanter, the femoral neck, and the epicondyles. For the pelvis, the iliac crest, the pubis, and the inferior and superior ilia were all readily accessible.

US images were acquired in two-dimensional compounding mode ('SonoCT' with the level of compounding set to "Survey") with a maximum penetration depth of $6 \mathrm{~cm}$ and a single focal zone. Before images were acquired, the level of the focal zone was adjusted to approximately correspond to the average depth of bone surface in order to maximize the image resolution at that depth. In compound mode, a number of images are formed by electronically steering the US beam through several preset angles. These images are then combined to produce a single compounded image. Compounding reduces speckle artefacts, but in this application it also had the useful effect of improving the echo intensity from regions of a curved bone surface not perpendicular to the axial direction of the image.

For the purpose of this study, bone surfaces were segmented manually from the captured US images using custom software written using Matlab. With this software, points lying on the bone surface were selected on the maximum intensity of the edge corresponding to bone-soft-tissue interface. A cubic spline was then fitted to these points to approximate the surface within the image slice so that an arbitrary number of bone surface points could be generated for each image. An average of 10 points were used per image.

\section{E. Reference 3D-US Calibration}

The reference 3D-US system calibration procedure used a point-target phantom and method previously described in [64]. Briefly, calibration involved capturing from many different views tracked images of a pinhead immersed in a water-glycerol solution. Seventy-six images were acquired in total, of which 71 were used to calculate a reference calibration transformation. Five images were excluded because either the pinhead was not visible or the tracking data was inaccurate, for example, because the US probe was moved during image capture. Exclusion of images with poor tracking data involved performing a calibration, reconstructing the $3-\mathrm{D}$ positions of the pinhead, and removing points that appeared as gross outliers.

The calibration procedure took approximately $90 \mathrm{~min}$ in total. Most this time ( $\sim 70 \mathrm{~min}$ ) was taken up carefully positioning the scan-probe and acquiring single images of the phantom. In the remaining time, the coordinates of the pinhead in each image were identified manually using custom-written software and the pixel scaling determined from electronic calliper measurements provided by the US scanner. The calibration transformation was computed very quickly ( $\sim 1 \mathrm{~s}$ ) using Matlab (The Mathworks Inc., Natick, MA).

The final RMS residual error in locating the pinhead was 1.1 $\mathrm{mm}$. The localization accuracy using this calibration method was found to be $0.6 \mathrm{~mm}$. This was estimated by comparing the 3-D positions, determined using the 3D-US system, of the centers of $1 \mathrm{~mm}$ diameter ball-bearings scanned in a water bath with those found using the digitizer with a $1 \mathrm{~mm}$ ball-tip attached.

\section{F. Point-to-Surface Registration Algorithm}

The algorithm implemented to register US-derived bone surface points to the CT-extracted bone surface is similar to that described by Fitzgibbon [65] in that a generalized nonlinear least-squares optimization scheme is employed to directly find the parameter vector, $\psi$, that minimizes the cost function, $C$, of the form

$$
C(\boldsymbol{\psi})=\sum_{l=1}^{l} \sum_{n=1}^{N} d^{2}\left(\mathbf{T}_{i}(\boldsymbol{\psi}) \cdot\left(\mathbf{p}_{n, i}\right)_{\mathrm{US}}, S\right)
$$

where $S$ is the CT-derived surface and $\left(\mathbf{p}_{n, i}\right)_{\mathrm{US}}=[u, v, 0,1]^{\mathrm{T}}$ is a (homogeneous) position vector that defines the $3-\mathrm{D}$ coordinates (in pixels) of the $n$th bone surface point derived from the $i$ th US image. $d(\mathbf{p}, S)$ returns the Euclidean distance between $S$ and the point, p. The 4-by-4 homogeneous transformation matrix, $\mathbf{T}_{i}(\psi)$, in (1) is defined as

$$
\begin{array}{r}
\mathbf{T}_{i}(\boldsymbol{\psi})=\underset{\mathrm{CT} \leftarrow \mathrm{DRO}}{\mathbf{T}}\left(\boldsymbol{\psi}_{\mathrm{REG}}\right) \cdot \underset{(\mathrm{OPT} \leftarrow \mathrm{DRO}, i)}{\mathbf{T}^{-1}} \cdot \underset{(\mathrm{OPT} \leftarrow \mathrm{PROBE}, i)}{\mathbf{T}} \\
\cdot \underset{\mathrm{PROBE} \leftarrow \mathrm{US}}{\mathbf{T}}\left(\boldsymbol{\psi}_{\mathrm{CAL}}\right) \cdot \mathbf{T}_{\mathrm{SCALE}}\left(s_{x}, s_{y}\right)
\end{array}
$$

where the subscripts "US," "PROBE," "OPT," and "DRO” denote the 3-D coordinate systems of the US image, the position sensor attached to the US scan-probe, the camera head of the optical localizer, and the DRO, respectively (see Fig. 1). The parameter vector, $\boldsymbol{\psi}=\left[\boldsymbol{\psi}_{\mathrm{REG}}, \boldsymbol{\psi}_{\mathrm{CAL}}, s_{x}, s_{y}\right]$, contains 6 rotations, 6 translations, and 2 scaling parameters.

The transformation matrices $\underset{\mathrm{OPT}}{\stackrel{\mathrm{T}}{\leftarrow \mathrm{DRO}, i}}$ and $\underset{\mathrm{OPT}}{\stackrel{\mathrm{PROBE}}{\mathrm{P}}, i}$ are calculated from measurements made by the optical localizer, and specify the 3-D position and orientation of the DRO 
and US probe relative to the coordinate system of the localizer, respectively. The subscript $i$ is used to explicitly denote a dependency on the image index. The matrix $\underset{\mathrm{PROBE} \leftarrow \mathrm{US}}{\mathbf{T}}$ in (2) is the rigid-body calibration matrix for the 3D-US system, and $\mathbf{T}_{\mathrm{SCALE}}$ is a diagonal scaling matrix defined as

$$
\mathbf{T}_{\text {SCALE }}=\left[\begin{array}{cccc}
s_{x} & 0 & 0 & 0 \\
0 & s_{y} & 0 & 0 \\
0 & 0 & 1 & 0 \\
0 & 0 & 0 & 1
\end{array}\right]
$$

where $s_{x}$ and $s_{y}$ are scaling factors (in $\mathrm{mm} / \mathrm{pixel}$ ) for the lateral and axial directions of the US image, respectively.

As explained in Section I, the calibration parameters (including pixel scaling) are normally determined preoperatively by performing a phantom-based calibration, with the resulting transformation matrices remaining constant during registration. However, here we assume that these parameters can be optimized by including them in $\boldsymbol{\psi}$. Then, given reasonable estimates of the values of all of the parameters in $\boldsymbol{\psi}$, a simultaneous registration and calibration can be performed by minimizing $C$ as defined in (1). In this work, $C$ was minimized using a trust-region implementation of the iterative Gauss-Newton algorithm provided in the Matlab Optimization Toolbox (v. 3.0). ${ }^{1}$ Since the optimized parameters comprise translations and rotations with different units, each parameter was scaled by the inverse of the magnitude of the corresponding column vector of the Jacobian, as described by Prager et al. [62], in order to ensure a well-conditioned system of equations. On each iteration of the algorithm, US-point-to-CT-surface distances were found efficiently to sub-voxel precision using trilinear interpolation of a precomputed 3-D Euclidean distance transform of the CT bone surface.

To avoid problems with convergence to local minima, the registration algorithm outlined above was adapted to execute as a 3 -step algorithm as follows: Firstly, a conventional rigid-body registration was performed with the calibration parameters held constant at their initial (reference) values-i.e. $\psi_{\mathrm{CAL}}=\psi_{\mathrm{CAL} 0}$, $s_{x}=s_{x 0}, s_{y}=s_{y 0}$ where $\boldsymbol{\psi}_{\mathrm{CAL} 0}, s_{x 0}$, and $s_{y 0}$ were determined using the phantom calibration described in Section II-E. This step provided an improved initial estimate of the registration parameters, $\psi_{\mathrm{REG} 1}$. Secondly, outliers were automatically removed by transforming the US points using the updated registration transformation, defined by $\psi_{\mathrm{REG} 1}$, and removing $10 \%$ of the points furthest from the bone surface. This method makes no assumptions about the distribution of point-to-surface distances and had the advantage that it was very simple to implement. Once outliers had been removed, a second rigid-body registration was performed using the remaining US points with $\boldsymbol{\psi}=\psi_{\mathrm{REG1}}$ as the starting registration parameters to yield a second updated set of registration parameters, $\boldsymbol{\psi}_{\mathrm{REG} 2}$. Finally, using $\boldsymbol{\psi}=\left[\boldsymbol{\psi}_{\mathrm{REG} 2}, \boldsymbol{\psi}_{\mathrm{CAL} 0}, s_{x 0}, s_{y 0}\right]$ as starting values, the algorithm was run again, this time including calibration param-

\footnotetext{
${ }^{1}$ It is worth noting that the method adopted here differs fundamentally from the popular iterative closest point algorithm [28] in that a general nonlinear optimization algorithm is used to iteratively reduce $C$ rather than computing an intermediate closed-form solution to register US-derived points to the set of closest points on $S$.
}

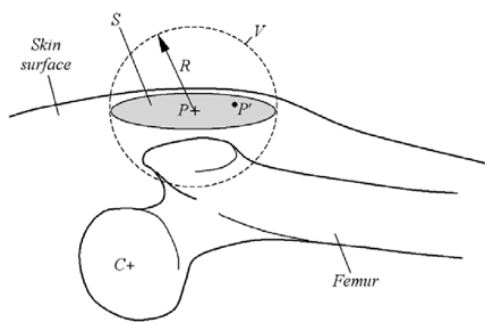

Fig. 4. Determination of landmark skin points used to calculate starting estimates for the physical-to-image registration transformation: a point, $P$, on the skin surface near to a palpable bony landmark (in this case the greater trochanter) is identified manually in the CT scan. A nearby point, $P^{\prime}$, is then selected at random on the shaded region of skin surface, $S$, which is bounded by a sphere, $V$, centered at $P$ with radius $R=20 \mathrm{~mm}$. This point is then transformed to physical space using the Gold Standard image-to-physical transformation to produce a digitized skin point corresponding to the CT point, $P$. Three such skin points were simulated for the femur, and 4 for the pelvis (see text). In the case of the femur, the center of rotation of the femoral head, $C$, provided a fourth landmark point.

eters as free parameters in the optimization. In order to compare the effect of optimizing just the axial scaling parameter, $s_{y}$, (which is directly related to the average speed of sound) with optimizing all 8 calibration parameters, this last step was repeated once using identical starting conditions, first including $s_{y}$ as a single additional free parameter, and then optimizing all 8 calibrations at the same time as the 6 rigid-body registration parameters.

\section{G. Registration Starting Estimates}

Surgically realistic starting estimates for the registration parameters were generated by simulating a simple point-based registration procedure that uses skin points near to palpable bony landmarks and, in the case of the femur, the center of rotation of the femoral head. Since skin points obtained with a physical digitizer were not available, suitable points were simulated using the procedure illustrated in Fig. 4. Initially, a point, $P$, on the skin surface near to a bony landmark was identified manually in the CT scan. Next, a random point, $P^{\prime}$, on the (CT-extracted) skin surface, within a radius of $R=20 \mathrm{~mm}$ of $P$, was generated automatically to simulate a digitization error. The point, $P^{\prime}$, was then transformed to physical space (i.e., DRO coordinates) by applying the Gold Standard registration transformation. Three such skin points, $\left\{P_{1}^{\prime}, P_{2}^{\prime}, P_{3}^{\prime}\right\}_{\mathrm{DRO}}$, were simulated for the femur, near to the medial and lateral epicondyles, and to the greater trochanter. For the pelvis, 4 points, $\left\{Q_{1}^{\prime}, Q_{2}^{\prime}, Q_{3}^{\prime}, Q_{4}^{\prime}\right\}_{\mathrm{DRO}}$, were simulated, near to the left and right superior pubic ramus, and the anterior superior iliac spines.

The center of rotation of the femoral head was already available in DRO coordinates, having been determined experimentally by pivoting the leg (see Section II-C). A random offset vector with magnitude $\leq 10 \mathrm{~mm}$ was added to this point to produce the new point, $C^{\prime}$, simulating the error that might reasonably be expected when determining the center of rotation using the pivot method. The corresponding center of rotation, $C$, in CT coordinates was defined as the geometric center of the femoral head, identified manually. The value of $10 \mathrm{~mm}$ was chosen based on our experience from a separate cadaver experiment in which the distance between the center of rotation, determined using 
TABLE I

Predicted TREs For the GOLd STANDARD REGISTRATION BASED ON BONE-IMPLANTED FidUCIALS AND THE NUMBER OF ULTRASOUND IMAGES AND POINTS USED FOR REGISTRATION

\begin{tabular}{|c|c|c|c|c|c|c|c|}
\hline Cadaver & Bone & $\begin{array}{c}\text { FRE } \\
(\mathrm{mm} \text { RMS })\end{array}$ & $\begin{array}{l}\text { Predicted TRE } \\
\text { over whole bone } \\
\text { (mm RMS) }\end{array}$ & $\begin{array}{l}\text { Predicted TRE } \\
\text { over surgical ROI } \\
\text { (mm RMS) }\end{array}$ & $\begin{array}{c}\text { Number of } \\
\text { fiducials }\end{array}$ & $\begin{array}{c}\text { Number of US } \\
\text { images used for } \\
\text { registration } \\
\text { (images acquired) }\end{array}$ & $\begin{array}{l}\text { Number of } \\
\text { US-derived } \\
\text { points }\end{array}$ \\
\hline \multirow[t]{3}{*}{1} & Left Femur & 0.06 & 1.03 & 1.27 & 3 & $211(248)$ & 2013 \\
\hline & Right Femur & 0.15 & 1.04 & 1.39 & 3 & $124(261)$ & 1447 \\
\hline & Pelvis & 0.44 & 0.69 & 0.49 & 5 & $143(282)$ & 1573 \\
\hline \multirow[t]{3}{*}{2} & Left Femur & 0.47 & 0.84 & 1.25 & 4 & $149(257)$ & 960 \\
\hline & Right Femur & 0.63 & 1.22 & 1.70 & 4 & $342(557)$ & 3096 \\
\hline & Pelvis & 0.86 & 0.50 & 0.41 & 8 & $276(482)$ & 1978 \\
\hline \multirow[t]{3}{*}{3} & Left Femur & 0.39 & 0.69 & 0.79 & 4 & $375(518)$ & 4421 \\
\hline & Right Femur & 0.73 & 0.72 & 0.78 & 4 & $510(576)$ & 6112 \\
\hline & Pelvis & 0.86 & 0.56 & 0.50 & 5 & $316(342)$ & 3030 \\
\hline
\end{tabular}

pivoting, and the estimated center, identified manually in the CT image, was found to be $4.8 \pm 2.1 \mathrm{~mm}$ (mean \pm SD over 10 hip joints) using the same localization system, protocol, and software as the present study.

One-hundred random starting estimates of the physical-to-image registration transformation $[\underset{\mathrm{CT} \leftarrow \mathrm{DRO}}{\mathbf{T}}$ in (2)] were generated for each bone by computing the rigid-body transformation relating the simulated and manually identified $\mathrm{CT}$ points. Specifically, the transformations relating $\left\{P_{1}, P_{2}, P_{3}, C\right\}_{\mathrm{CT}}$ and $\left\{P_{1}^{\prime}, P_{2}^{\prime}, P_{3}^{\prime}, C^{\prime}\right\}_{\mathrm{DRO}}$, and $\left\{Q_{1}, Q_{2}, Q_{3}, Q_{4}\right\}_{\mathrm{CT}}$ and $\left\{Q_{1}^{\prime}, Q_{2}^{\prime}, Q_{3}^{\prime}, Q_{4}^{\prime}\right\}$ DRO, were determined for the femur and pelvis, respectively. The aim here was to produce initial registration parameters that were approximately representative of those that might be encountered using a simple, noninvasive protocol. Although effects such as skin deformation were not explicitly modeled in this procedure, setting the maximum error to $20 \mathrm{~mm}$ provided a pessimistic "worst case" upper bound on the error associated with manual digitization of palpated landmarks [66], for which additional errors introduced by factors such as skin deformation may be considered to be insignificant.

In order to test the robustness of the 3-step point-to-surface registration algorithm, 2 series of experiments were carried out: In the first, 100 registrations were performed using each of the random initial registration estimates, with the initial values for the calibration parameters set to those found using the reference calibration based on 71 images of a point-target phantom as described in Section II-E. In the second set of experiments, 100 "noisy" calibrations were simulated by randomly selecting 35 out of the 71 phantom images and adding uniform random noise $\leq 3 \mathrm{~mm}$ to the $x-, y$-, and $z$-coordinates of the pinhead, determined for each image. Uniformly distributed, random noise of $\pm 5 \%$ was also added to the value of the two image scaling parameters. Three calibrations were excluded on the grounds of a poor RMS residual error $(>5 \mathrm{~mm})$ in locating the point-target.

The accuracy of the noisy calibrations was estimated by computing the RMS distance between the 3-D positions of US image pixels, calculated using the noisy calibration transformations and the reference calibration based on the original data from 71 calibration images. The pixel positions chosen formed a regularly spaced grid of $5 \times 6$ points in each US image acquired on all 9 bones. Using this error metric, the estimated accuracy of the noisy calibration transformations was $2.29-\mathrm{mm}$ RMS $(\max =9.52 \mathrm{~mm})$.

US-to-CT registrations were performed on each femur and each complete pelvis with the assumption that the hemi-pelves were fused and, therefore, sufficiently rigid to be able to register the whole pelvis. This assumption was verified by the low fiducial registration error (FRE) presented later in Table I. As a measure to automatically exclude poor tracking data, bone surface points were only considered from US images for which $\geq 10$ out of 20 LEDs, and $\leq 5$ out of 6 LEDs were visible on the US probe tracker and DRO, respectively.

\section{H. Error Analysis}

Registration accuracy was evaluated by calculating the target registration error (TRE) for points on the bone surface in the CT scan using the formula

$\operatorname{TRE}\left(\mathbf{s}_{\mathrm{CT}}\right)=\left\|\left[(\underset{\mathrm{CT} \leftarrow \mathrm{DRO}}{\mathbf{T}})_{\mathrm{US}} \cdot(\underset{\mathrm{CT} \leftarrow \mathrm{DRO}}{\mathbf{T}})_{\mathrm{GOLD}}^{-1} \cdot \mathbf{S}_{\mathrm{CT}}\right]-\mathbf{s}_{\mathrm{CT}}\right\|$ where $\mathrm{S}_{\mathrm{CT}}$ is the homogeneous position vector of the center of a bone surface voxel (with respect to the CT coordinate system), and the subscripts 'US' and 'GOLD' denote transformations computed using US-based and Gold Standard registrations, respectively. (Note: In (4) the magnitude operator ignores the redundant 4th element of the homogeneous vector operand, i.e. $\left\|[x y z 1]^{\mathrm{T}}\right\|=\sqrt{x^{2}+y^{2}+z^{2}}$.)

For each registration, the RMS TRE was computed over all bone surface voxels as an overall measure of registration accuracy. As a further more clinically relevant measure, the RMS TRE was computed over the subset of voxels lying within a surgical region of interest (SROI). For the femur, the SROI was defined as the region proximal to the lesser trochanter, which included the femoral head and neck; for the pelvis, the SROI was defined as the spherical region enclosing the acetabulum with radius $50 \mathrm{~mm}$, centered at the centroid of the femoral head, manually identified in the $\mathrm{CT}$ image. 


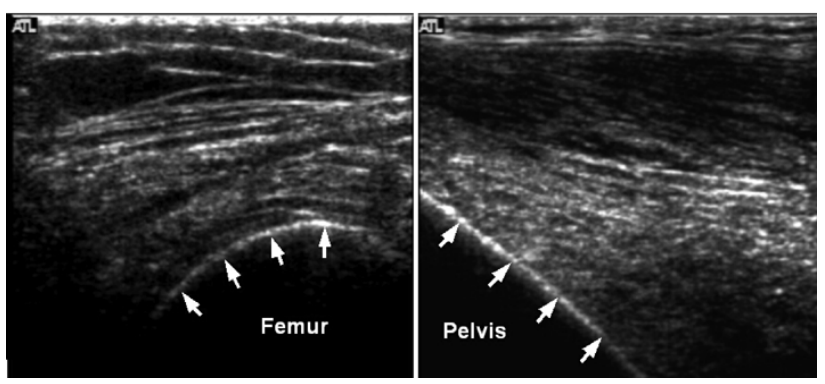

Fig. 5. Example US images of the femur and pelvis obtained from a cadaver. The arrows indicate the bone surface.

\section{RESULTS}

Between 248 and 576 tracked US images were acquired for each cadaveric femur and pelvis. Two examples are shown in Fig. 5. The number of images and sample points used for each bone are given in Table I. The mean depth of the bone surface was $18.2 \mathrm{~mm}(\max =45.3 \mathrm{~mm})$ for the femur and $16.6 \mathrm{~mm}$ $(\max =58.3)$ for the pelvis. For each bone, the fiducial registration error (FRE) and predicted TREs for the Gold Standard, calculated using bone-implanted fiducials, are summarized in Table I. The predicted TREs were calculated using the following formula described by Fitzpatrick et al. [67]:

$$
\left\langle\mathrm{TRE}^{2}\right\rangle \approx \frac{1}{N}\left(1+\frac{1}{3} \sum_{k=1}^{3} \frac{d_{k}^{2}}{f_{k}^{2}}\right)\left\langle\overline{\mathrm{FLE}}^{2}\right\rangle
$$

where $\left\langle\mathrm{TRE}^{2}\right\rangle$ is the expected squared TRE for a target point on the bone surface; $N$ is the number of bone-implanted fiducials; $d_{k}$ is the distance of the target point from the $k$ th principal axis of the fiducials; $f_{k}$ is the RMS distance of the fiducials from the same axis; and $\left\langle\overline{\mathrm{FLE}}^{2}\right\rangle$ is an estimate of the expected fiducial localization error given by [68]

$$
\left\langle\overline{\mathrm{FLE}}^{2}\right\rangle \approx \frac{1}{M} \sum_{m=1}^{M} \frac{N_{m} \cdot \mathrm{FRE}_{m}^{2}}{\left(N_{m}-2\right)}
$$

where $M=9$ is the total number of bones; $N_{m}$ is the number of fiducials for the $m$ th bone; and $\mathrm{FRE}_{m}$ is the RMS FRE calculated for the $m$ th bone (column 3 in Table I). Using this equation, the estimated RMS FLE was $0.76 \mathrm{~mm}$.

Inspection of Table I reveals that, in some cases, fewer fiducials were used to calculate the Gold Standard registration than the number originally implanted (4 for each femur and 10 for each pelvis). The reason for this was that some fiducials became loose during the course of the experiments, particularly those implanted in the pelvis where the cortical bone is thin. These fiducials were excluded to maximize the accuracy of the Gold Standard registrations.

In the least-squares optimization used to match US-derived points to the CT surface, scaling the free parameters by the magnitude of the column vectors of the Jacobian resulted in a well-conditioned system of equations, indicated by a condition number, defined as the ratio of the largest and smallest singular values of the Jacobian, $<20$ in all cases (the recommended limit of 100 is often applied [62]). The average time for performing a 3-step, self-calibrating registration using Matlab was approximately 100 s on a PC with a $1.3 \mathrm{GHz}$ AMD Athlon XP 1500+ processor and 2 GB RAM.

Tables II and III show the initial and final TREs (averaged over 100 registration trials) calculated for each bone using calibration parameters determined using the reference and noisy phantom calibrations, respectively. It can be seen from the results presented that, in most cases, excluding outliers improved the registration accuracy. Moreover, further improvements in the registration accuracy were obtained in nearly all cases by including calibration parameters into the registration optimization, with the largest reduction in TRE occurring when all 8 calibration parameters were optimized. In one case-Cadaver 2, right femur-including the calibration parameters increased the TRE over the whole bone surface, but it should be noted that this bone also had the poorest estimated Gold Standard registration (see Table I). Applying the Kolmogorov-Smirnov test revealed that the differences between TRE 2 and TREs 3 and 4 in Tables II and III were statistically significant at the $1 \%$ significance level.

Overall, the failure rate of the 3-step algorithm was low, with 48 failures occurring from 900 registrations when all calibration parameters were optimized. It can be seen from Tables II and III that 39 of these failures occurred when registering the pelvis of Cadaver 2, which we attribute to the relatively large initial TREs used for this bone. Failure rates were found to be higher when registering the pelves, and this is likely to be largely due to the poorer simulated starting estimates computed for these bones, which is reflected by the higher initial TREs compared with the femurs, combined with relatively noisy US point data.

The results in Table III indicate that the registrations were robust to noisy initial calibration parameters, with a relatively small difference in the overall accuracy and the number of failures compared with using a high-quality preoperative calibration (Table II).

\section{DISCUSSION}

The invasiveness of conventional, anatomy-based methods for registering bones seriously compromises the minimally invasive advantage otherwise offered by CAOS. Furthermore, since registration accuracy increases as the number and spatial distribution of digitized points increases [67], [69], the surgeon is presented with a difficult problem, which in practice dictates that a compromise must be reached between the level of invasiveness acceptable and the navigational accuracy that can be expected.

Percutaneous localization of the bone surface using freehand 3D-US is a highly attractive alternative to conventional methods because it overcomes the need for exposing the bone surface or implanting fiducial markers, which are associated with the most accurate methods conventionally used for bone registration. The technique also makes use of 3-D localization devices already utilized by modern surgical navigation systems. It should be noted, however, that implantation of a DRO is still necessary, but this is currently standard practice during CAOS and can be performed using a small incision. One further advantage of US-based registration, particularly when B-mode imaging is employed, is that many more surface 
TABLE II

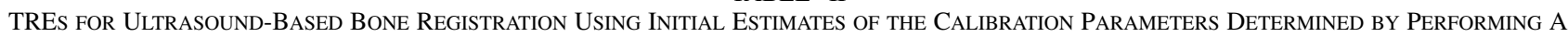
REFERENCE PHANTOM CALIBRATION BASED on 71 IMAges of A Point-TARget

\begin{tabular}{|c|c|c|c|c|c|c|c|c|}
\hline Cadaver & Bone & Region & Initial TRE & $\operatorname{TRE} 1^{\mathrm{a}}$ & $\operatorname{TRE} 2^{\mathrm{b}}$ & $\operatorname{TRE} 3^{\mathrm{c}}$ & $\operatorname{TRE} 4^{\mathrm{d}}$ & $\begin{array}{l}\text { Failures for } \\
\text { registrations } 1-4\end{array}$ \\
\hline \multirow[t]{2}{*}{1} & Left Femur & Whole Bone & $8.08(22.27)$ & $1.82(2.89)$ & $1.67(2.66)$ & $1.48(2.03)$ & $0.96(2.03)$ & $0-0-0-0$ \\
\hline & & Surgical ROI & $7.74(18.71)$ & $1.52(2.27)$ & $1.40(1.84)$ & $1.18(1.67)$ & $0.74(1.36)$ & \\
\hline \multirow[t]{2}{*}{1} & Right Femur & Whole Bone & $7.86(20.43)$ & $0.81(2.22)$ & $0.74(1.63)$ & $0.71(1.46)$ & $0.36(1.33)$ & $0-0-0-0$ \\
\hline & & Surgical ROI & $7.66(20.43)$ & $0.67(1.33)$ & $0.57(1.05)$ & $0.56(0.93)$ & $0.23(0.59)$ & \\
\hline \multirow[t]{2}{*}{1} & Pelvis & Whole Bone & $11.48(31.75)$ & $4.79(8.48)$ & $1.83(6.83)$ & $1.72(6.82)$ & $1.76(5.02)$ & $21-1-1-6$ \\
\hline & & Surgical ROI & $9.71(22.94)$ & $3.65(6.14)$ & $1.83(4.72)$ & $1.78(4.68)$ & $1.93(4.17)$ & \\
\hline \multirow[t]{2}{*}{2} & Left Femur & Whole Bone & $8.13(20.44)$ & $1.71(3.21)$ & $1.84(3.15)$ & $1.25(2.49)$ & $1.14(2.37)$ & $0-0-0-0$ \\
\hline & & Surgical ROI & $8.00(18.74)$ & $2.41(3.21)$ & $2.44(3.15)$ & $1.78(2.49)$ & $1.79(2.37)$ & \\
\hline \multirow[t]{2}{*}{2} & Right Femur & Whole Bone & $7.97(20.80)$ & $3.34(5.73)$ & $2.23(5.21)$ & $2.53(5.21)$ & $2.35(4.54)$ & $17-2-0-0$ \\
\hline & & Surgical ROI & $7.18(18.22)$ & $3.57(5.59)$ & $2.66(5.21)$ & $2.70(4.93)$ & $2.44(4.53)$ & \\
\hline \multirow[t]{2}{*}{2} & Pelvis & Whole Bone & $20.07(64.88)$ & $3.57(6.14)$ & $2.74(4.32)$ & $2.41(4.01)$ & $1.98(3.77)$ & $39-39-39-39$ \\
\hline & & Surgical ROI & $14.83(39.22)$ & $3.47(5.99)$ & $2.59(3.53)$ & $2.29(3.21)$ & $2.01(3.55)$ & \\
\hline \multirow[t]{2}{*}{3} & Left Femur & Whole Bone & $7.95(25.97)$ & $2.41(3.59)$ & $2.52(4.09)$ & $2.09(3.56)$ & $1.67(2.59)$ & $0-0-0-0$ \\
\hline & & Surgical ROI & $7.50(22.32)$ & $2.61(3.59)$ & $2.51(4.09)$ & $2.07(3.00)$ & $1.76(2.53)$ & \\
\hline \multirow[t]{2}{*}{3} & Right Femur & Whole Bone & $8.07(13.09)$ & $1.82(3.16)$ & $1.83(3.03)$ & $1.52(2.60)$ & $1.25(2.63)$ & $0-0-0-0$ \\
\hline & & Surgical ROI & $8.14(19.46)$ & $2.62(3.16)$ & $2.54(3.03)$ & $2.12(2.60)$ & $1.99(2.63)$ & \\
\hline \multirow[t]{2}{*}{3} & Pelvis & Whole Bone & $12.67(35.83)$ & $3.53(5.76)$ & $3.71(5.90)$ & $2.95(4.94)$ & $2.78(4.57)$ & $3-3-3-3$ \\
\hline & & Surgical ROI & $10.89(27.34)$ & $3.64(5.46)$ & $3.82(5.60)$ & $2.99(4.53)$ & $2.76(4.23)$ & \\
\hline \multirow[t]{2}{*}{ All } & All & Whole Bone & $10.25(64.88)$ & $2.53(8.48)$ & $2.09(6.83)$ & $1.82(6.82)$ & $1.56(5.02)$ & $80-45-43-48$ \\
\hline & & Surgical ROI & $9.07(39.22)$ & $2.60(6.14)$ & $2.24(5.60)$ & $1.92(4.93)$ & $1.72(4.53)$ & \\
\hline
\end{tabular}

All TREs are quoted as the average of 100 RMS TREs (in $\mathrm{mm}$ ) corresponding to 100 registration trials, with the absolute maximum TRE given in parentheses.

${ }^{\mathrm{a}}$ TRE $1=$ TRE following an initial rigid registration with fixed calibration parameters (Algorithm Step 1).

${ }^{\mathrm{b}}$ TRE $2=$ TRE following a repeated rigid registration for which outliers were removed (Algorithm Step 2).

${ }^{\mathrm{c}}$ TRE $3=$ TRE following a rigid registration where the axial pixel scaling of the ultrasound image $\left(s_{y}\right)$ was included in the registration optimization (Algorithm Step 3).

${ }^{\mathrm{d}}$ TRE $4=$ TRE following a rigid registration where all 8 calibration parameters were included in the registration optimization (Algorithm Step 3)

${ }^{\mathrm{e}}$ Registration failure was defined as TRE $>5.00 \mathrm{~mm}$ RMS

TABLE III

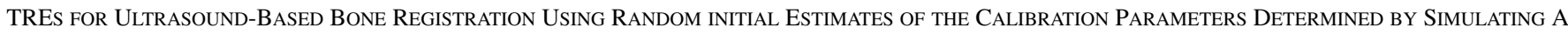
Noisy Phantom Calibration Based on 35 Images of A Point-Target (See TeXt For Details)

\begin{tabular}{|c|c|c|c|c|c|c|c|c|}
\hline Cadaver & Bone & Region & Initial TRE & $\operatorname{TRE} 1^{\mathrm{a}}$ & $\operatorname{TRE} 2^{\mathrm{b}}$ & $\operatorname{TRE} 3^{\mathrm{c}}$ & TRE $4^{\mathrm{d}}$ & $\begin{array}{l}\text { Failures for } \\
\text { registrations } 1-4^{\mathrm{e}}\end{array}$ \\
\hline \multirow[t]{2}{*}{1} & Left Femur & Whole Bone & $8.08(22.27)$ & $1.92(5.23)$ & $1.79(4.61)$ & $1.51(3.42)$ & $1.00(2.73)$ & $0-0-0-0$ \\
\hline & & Surgical ROI & $7.74(18.71)$ & $1.74(4.33)$ & $1.66(4.17)$ & $1.31(3.10)$ & $0.81(1.89)$ & \\
\hline \multirow[t]{2}{*}{1} & Right Femur & Whole Bone & $7.86(20.43)$ & $1.09(3.50)$ & $1.07(3.30)$ & $1.04(3.21)$ & $0.54(1.98)$ & $0-0-0-0$ \\
\hline & & Surgical ROI & $7.66(20.43)$ & $1.02(3.26)$ & $0.98(3.30)$ & $0.90(3.21)$ & $0.36(1.14)$ & \\
\hline \multirow[t]{2}{*}{1} & Pelvis & Whole Bone & $11.48(31.75)$ & $4.63(9.20)$ & $2.20(7.31)$ & $2.05(8.35)$ & $2.02(8.36)$ & $35-2-0-6$ \\
\hline & & Surgical ROI & $9.71(22.94)$ & $3.63(6.86)$ & $2.12(5.58)$ & $1.99(6.00)$ & $2.12(5.63)$ & \\
\hline \multirow[t]{2}{*}{2} & Left Femur & Whole Bone & $8.13(20.44)$ & $2.01(5.74)$ & $2.14(5.99)$ & $1.43(4.32)$ & $1.09(2.55)$ & $0-0-0-0$ \\
\hline & & Surgical ROI & $8.00(18.74)$ & $2.65(5.74)$ & $2.66(5.99)$ & $1.87(4.32)$ & $1.69(2.55)$ & \\
\hline \multirow[t]{2}{*}{2} & Right Femur & Whole Bone & $7.97(20.80)$ & $3.86(9.10)$ & $3.10(9.55)$ & $2.76(5.25)$ & $2.53(5.24)$ & $22-3-2-2$ \\
\hline & & Surgical ROI & $7.18(18.22)$ & $4.24(9.10)$ & $3.62(9.55)$ & $2.97(5.25)$ & $2.63(5.24)$ & \\
\hline \multirow[t]{2}{*}{2} & Pelvis & Whole Bone & $20.07(64.88)$ & $3.56(7.33)$ & $2.71(7.82)$ & $2.47(6.40)$ & $1.99(6.20)$ & $38-36-36-36$ \\
\hline & & Surgical ROI & $14.83(39.22)$ & $3.46(6.67)$ & $2.56(6.23)$ & $2.35(6.04)$ & $2.01(5.04)$ & \\
\hline \multirow[t]{2}{*}{3} & Left Femur & Whole Bone & $7.95(25.97)$ & $2.54(5.24)$ & $2.64(6.62)$ & $2.15(5.26)$ & $1.67(2.78)$ & $0-0-0-0$ \\
\hline & & Surgical ROI & $7.50(22.32)$ & $2.74(4.85)$ & $2.70(6.17)$ & $2.18(3.94)$ & $1.77(2.78)$ & \\
\hline \multirow[t]{2}{*}{3} & Right Femur & Whole Bone & $8.07(20.99)$ & $1.97(4.93)$ & $1.99(4.94)$ & $1.66(4.30)$ & $1.27(2.71)$ & $0-0-0-0$ \\
\hline & & Surgical ROI & $8.14(19.46)$ & $2.72(4.93)$ & $2.67(4.94)$ & $2.24(4.30)$ & $2.01(2.71)$ & \\
\hline \multirow[t]{2}{*}{3} & Pelvis & Whole Bone & $12.67(35.83)$ & $3.66(7.43)$ & $3.78(7.28)$ & $3.05(5.85)$ & $2.75(4.85)$ & $6-11-4-4$ \\
\hline & & Surgical ROI & $10.89(27.34)$ & $3.76(7.12)$ & $3.88(7.04)$ & $3.09(5.54)$ & $2.73(4.56)$ & \\
\hline \multirow[t]{2}{*}{ All } & All & Whole Bone & $10.25(64.88)$ & $2.65(9.20)$ & $2.35(9.55)$ & $1.99(8.35)$ & $1.63(8.36)$ & $101-52-42-48$ \\
\hline & & Surgical ROI & $9.07(39.22)$ & $2.78(9.10)$ & $2.52(9.55)$ & $2.08(6.04)$ & $1.77(5.63)$ & \\
\hline
\end{tabular}

All TREs are quoted as the average of 100 RMS TREs (in $\mathrm{mm}$ ) corresponding to 100 registration trials, with the absolute maximum TRE given in parentheses. ${ }^{a-e}$ See Table II 
points can be acquired than would be possible with manual digitization by direct contact. Therefore, registration accuracy is also potentially increased and the sample time reduced.

A number of researchers have already investigated the accuracy of US-based bone registration using phantoms, with reported errors comparable to, if not better than anatomy-based registration techniques. Importantly, however, very few validation studies are reported in the literature which make use of clinically realistic US data. One exception is Amin et al. [47] who report an average RMS registration error of $1.27 \mathrm{~mm}$ in translation and $0.59^{\circ}$ in rotation for the pelvis compared with a commercially available technique (the 'HipNav' protocol [2]). The corresponding values calculated for the data obtained in our study are: $1.90 \mathrm{~mm}$ and $0.48^{\circ}$ for the pelvis, and 1.15 $\mathrm{mm}$ and $0.33^{\circ}$ for the femur (based on the data corresponding to Table III, where initial calibration estimates based on low quality data were used). Some caution should be exercised when interpreting these values since the translational and rotational parameters of a rigid-body registration are not independent and, therefore, such error metrics can give a misleading impression of registration accuracy. For this reason, the TRE is a preferable measure of registration accuracy. The data presented from the study by Amin et al. is also limited to the registration of a single patient hemi-pelvis, repeated 10 times, and the accuracy of the Gold Standard registration method was not reported.

To date, no previous studies have adequately addressed the significant localization errors introduced by variations in acoustic properties of soft tissue surrounding bone, in particular, deviations from the assumed average speed of sound of $1540 \mathrm{~m} / \mathrm{s}$. The self-calibration approach introduced in this paper allows compensation for such effects by assuming that, for a given set of US images, a significant component of the resulting registration error can be modeled as an unknown error in the 3D-US calibration parameters. This assumption was confirmed by the improvement in registration accuracy observed if calibration parameters are included in the registration optimization. The large amount of sample data used to determine the updated calibration parameters compared with the reference phantom calibration method (Section II-E) is also likely to be a significant factor in the improved accuracy of the these parameters.

In the 3D-US system used in this study, the position sensor and scan-probe attachments were designed so that the sensor could be precisely relocated into a holder permanently attached to the scan probe. This in itself reduces the need for recalibration using a phantom, but, in common with diagnostic US systems, there is an argument that calibration should still be performed regularly as a quality assurance procedure, since the characteristics of system components, such as the position sensor, can change with use over time.

This is not particularly time-consuming if a rapid phantom calibration technique is employed (e.g. [61], [62], [70]); for example, Pagoulatos et al. [70] report a calibration time of approximately 2 min using a Z-shaped wire phantom. However, the robustness of the self-calibrating registration algorithm to initial estimates of calibration parameters, based on low quality, noisy calibration data, suggests that routine highly accurate recalibra- tion of the 3D-US system using a phantom may not be necessary unless the calibration parameters are expected to have changed significantly between scans, for example, if the holder for the position sensor is moved to a new position. Intraoperative adjustments of the US scanning settings for individual patients are also possible by storing estimates of the image scaling parameters corresponding to a number of different depth settings in a lookup table, and updating these values during the registration. Intraoperative adjustment of other parameters, such as the level and number of focal zones, which preserve image size but might have a nonnegligible effect on the calibration parameters (for instance, due to changes in US beam thickness), are also possible using self-calibrating registration. In contrast, such changes to the imaging parameters might warrant a full recalibration using a phantom using the conventional fixed calibration parameter approach.

One important prerequisite for accurate self-calibration is that, in common with phantom-based calibration techniques, US images should be obtained from a wide range of view directions and positions in order to produce a well-conditioned system of equations [61], [62]. This requirement is also conducive to accurate registration, and, from our experience in this study, we did not find it difficult to obtain hundreds of US images of the femur and pelvis from a large range of positions and orientations. The time taken to acquire images in this study ranged from approximately 5 to $30 \mathrm{~min}$ in total. These durations, which are longer than would be acceptable in clinical practice, should be interpreted in the context of the aims of this study. In particular, although a small amount of preliminary work had been carried out using volunteers, an optimal scanning protocol was not available and we had relatively little idea how much bone surface was accessible in the surgical situation. Therefore, a significant amount of time was spent investigating this issue, carefully imaging the bone surface in as many different locations as possible. We anticipate that the time taken to acquire images for registration could be reduced to within $5 \mathrm{~min}$ by developing an optimal protocol that ensures images are obtained from a sufficient range of views to ensure accurate registration and self-calibration. This is the subject of future work.

The use of cadavers provided an indication of the regions of bone surface accessible to US during orthopedic procedures. This was potentially problematic because, taking the example of the femur, although the femoral shaft should be easy to image for most patients, it has relatively few unique geometrical features. This implies that points from additional, less easy-toimage regions at the extremities of the bone, should be included to better constrain the translational and rotational components of the registration transformation in and about the long axis of the femur. Around femoral head and neck, which is a surgically important region in THR, the presence of tendons and ligaments, through which US is unable to penetrate, and occlusion by the acetabulum, prevent this region from being well-sampled, especially in the obese patient where the penetration depth of the US probe may be inadequate. In practice, however, our initial experience is that sufficient portion of the surface of both the femur and pelvis can be imaged using US to enable accurate registra- 
tion of the whole bone and the region of interest most relevant to THR. This is a significant finding in itself, which, to the best of our knowledge, has not been demonstrated previously for the pelvis and femur using a realistic anatomical model and accurate Gold Standard registration transformations.

The simulated intraoperative registration procedure based on skin points provided sufficiently accurate starting estimates in most cases. More accurate starting estimates could be obtained for registering the pelvis by selecting additional landmark points. An obvious extension to the protocol already described would be the inclusion of the center of rotation of the femoral head as an additional point. It should be borne in mind, however, that, in general, estimating the location of this point by pivoting the leg is possible only when a DRO has been implanted into the femur, which may not be justified for procedures where surgical guidance is only required on the pelvis. Conversely, for procedures that require guidance only on the femur, a reasonable estimate of the center of rotation may be obtained by assuming (as in this study) that the pelvis is immobile during pivoting and avoiding extreme leg movements that could compromise this assumption.

The algorithm described in this paper requires prior segmentation of bone surface points from US images. In order to maximize accuracy, manual point extraction was adopted, which would not be practical for a clinical system due to the large time overhead associated with this procedure. Accurate and automatic segmentation of the bone surface from US images is not addressed in this paper, which is concerned with the presentation and rigorous validation of a new self-calibrating approach to bone registration. A number of potential solutions to the segmentation problem have been recently proposed, any of which could provide input data for the algorithm without modification [47], [71]-[73]. It should be noted, however, that the accuracy of the technique described here relies on accurate segmentation of the bone surface, which is dependent on the US scanner and scan-probe used, as well as the segmentation method employed. In this study, we used a state-of-the art scanner and a high frequency transducer in conjunction with realtime compounding to maximize visualization of the bone surface. Currently, this solution is too expensive for dedicated clinical use in this application. Furthermore, a lower frequency probe with greater penetration depth, but lower axial resolution, may be more appropriate to image bone surfaces in a wide range of the patient population. Nevertheless, we anticipate that, given current trends in US transducer technology, electronics and signal processing, it is feasible to manufacture a low-cost, dedicated US device specifically for the purpose of noninvasive bone registration, which provides accuracy sufficient for clinical use.

A further important issue is the treatment of outliers in the US pointset. We used a very simple method in which outliers were defined as the furthest $10 \%$ of points from the bone surface. Although this method was very easy to implement, potentially useful, nonoutlying points may be discarded. More sophisticated outlier removal schemes may be more appropriate, especially if the number of US points is reduced, for example, by adopting a clinical protocol where the number of US images is reduced to minimize the intraoperative scan time. One approach, which would be a straightforward extension of the algorithm outlined here, would be to incorporate robust estimation by applying a Lorentzian or Huber function to the distance transform as described by Fitzgibbon [65].

An alternative approach to the registration problem, which is the subject of recent attention from our research group, is the development of an automatic, image-intensity-based method for bone registration using US data. This approach has the advantage that the bone surface does not need to be explicitly segmented. Future work will investigate methods for incorporating the concept of self-calibration demonstrated in this paper into an intensity-based registration scheme, as well as developing a practical clinical system and protocol for intraoperative use.

\section{CONCLUSION}

We conclude that bone registration using a self-calibrating 3D-US-based algorithm provides a noninvasive and accurate method for registering bones during image-guided orthopedic procedures. Using realistic US images obtained on cadavers, and under conditions representative of those encountered during THR surgery, we found the self-calibrating algorithm resulted in a reduction in TRE of approximately $30 \%$, compared with a standard point-based algorithm where the calibration parameters for the freehand 3D-US system were fixed to values determined using a preoperative phantom calibration. Therefore, this method provides a promising means of compensating for some of the significant sources of error inherent in conventional US-based registration techniques, which rely exclusively on a phantom-based calibration.

\section{ACKNOWLEDGMENT}

The authors would like to thank Prof. R. Putz at the Anatomische Anstalt, LMU, Munich, Germany, for providing cadavers and experimental facilities for this study; the staff in the Radiology Department at LMU for performing CT scans; J. Krugman of BrainLab AG, Munich, Germany, for his assistance in organizing the cadaver experiments; and A. Black in the Medical Physics workshop at Guy's Hospital, King's College London, for making the tracked attachments for the US probe used in this study.

\section{REFERENCES}

[1] L.-P. Amiot and F. Poulin, "Computed tomography-based navigation for hip, knee, and spine surgery," Clin. Orthop., vol. 421, pp. 77-86, 2004.

[2] A. M. DiGioia, B. Jaramaz, M. Blackwell, D. A. Simon, F. Morgan, J. E. Moody, C. Nikou, B. D. Colgan, C. A. Aston, R. S. LaBarca, E. Kischell, and T. Kanade, "The Otto Aufranc Award. Image guided navigation system to measure intraoperatively acetabular implant alignment," Clin. Orthop., vol. 355, pp. 8-22, 1998.

[3] A. M. DiGioia III and L. P. Nolte, "The challenges for CAOS: what is the role of CAOS in orthopaedics?," Comput. Aided Surg., vol. 7, pp. 127-128, 2002.

[4] P. Noble, N. Sugano, J. Johnson, M. Thompson, M. Conditt Sr., C. Engh, and K. Mathis, "Computer simulation: how can it help the surgeon optimize implant position," Clin. Orthop., vol. 417, pp. 242-252, 2003.

[5] S. Stulberg and T. Kienzle, "Computer- and robot-assisted orthopaedic surgery," in Computer Integrated Surgery: Technology and Clinical Applications, R. H. Taylor, S. Lavallée, G. C. Burdea, and R. Mösges, Eds. Cambridge, MA: MIT Press, 1996, pp. 373-378. 
[6] A. M. DiGioia III, A. Y. Plakseychuk, T. J. Levison, and B. Jaramaz, "Mini-incision technique for total hip arthroplasty with navigation," $J$. Arthroplasty, vol. 18, pp. 123-128, 2003.

[7] A. M. DiGioia III, B. Jaramaz, A. Y. Plakseychuk, J. E. Moody, Jr., C. Nikou, R. S. LaBarca, T. J. Levison, and F. Picard, "Comparison of a mechanical acetabular alignment guide with computer placement of the socket," J. Arthroplasty, vol. 17, pp. 359-364, 2002.

[8] B. Jaramaz, A. M. DiGioia III, M. Blackwell, and C. Nikou, "Computer assisted measurement of cup placement in total hip replacement," Clin. Orthop., vol. 354, pp. 70-81, 1998.

[9] K.-H. Widmer and P. A. Grützner, "Joint replacement-total hip replacement with CT-based navigation," Injury, vol. 35, pp. 84-89, 2004.

[10] R. H. Taylor, L. Joskowicz, B. Williamson, A. Gueziec, A. Kalvin, P. Kazanzides, R. Van Vorhis, J. Yao, R. Kumar, A. Bzostek, A. Sahay, M. Borner, and A. Lahmer, "Computer-integrated revision total hip replacement surgery: concept and preliminary results," Med. Image Anal., vol. 3, pp. 301-319, 1999.

[11] J. Tonetti, L. Carrat, S. Lavallee, L. Pittet, P. Merloz, and J. P. Chirossel, "Percutaneous iliosacral screw placement using image guided techniques," Clin. Orthop., vol. 354, pp. 103-110, 1998.

[12] J. Herring, B. Dawant, C. Maurer, D. Muratore, R. Galloway, and M. Fitzpatrick, "Surface-based registration of CT images to physical space for image-guided surgery of the spine: a sensitivity study," IEEE Trans. Med. Imag., vol. 17, no. 5, pp. 743-752, Oct. 1998.

[13] F. Langlotz, R. Bachler, U. Berlemann, L. P. Nolte, and R. Ganz, "Computer assistance for pelvic osteotomies," Clin. Orthop., vol. 354, pp. 92-102, 1998.

[14] R. E. Ellis, C. Y. Tso, J. F. Rudan, and M. M. Harrison, "A surgical planning and guidance system for high tibial osteotomy," Comput. Aided Surg., vol. 4, pp. 264-274, 1999.

[15] G. S. Athwal, R. E. Ellis, C. F. Small, and D. R. Pichora, "Computer-assisted distal radius osteotomy," J. Hand Surg. [Am.], vol. 28, pp. 951-958, 2003.

[16] J. E. Moody, C. Nikou, F. Picard, T. Levison, B. Jaramaz, A. M. DiGioia III, and C. F. Reverte, "Computer-integrated anterior cruciate ligament reconstruction system," J. Bone Joint Surg. Am. (Suppl. 2), vol. 84-A, pp. 99-101, 2002.

[17] F. Picard, A. M. DiGioia, J. Moody, V. Martinek, F. H. Fu, M. Rytel, C. Nikou, R. S. LaBarca, and B. Jaramaz, "Accuracy in tunnel placement for ACL reconstruction. Comparison of traditional arthroscopic and computer-assisted navigation techniques," Comput. Aided Surg., vol. 6, pp. 279-289, 2001.

[18] G. S. Athwal, D. R. Pichora, R. E. Ellis, and J. F. Rudan, "A computerassisted guidance technique for the localization and excision of osteoid osteoma," Orthopedics, vol. 27, pp. 195-197, 2004.

[19] H. Handels, J. Ehrhardt, W. Plötz, and W. Pöppl, “Three-dimensional planning and simulation of hip operations and computer-assisted construction of endoprostheses in bone tumor surgery," Comput. Aided Surg., vol. 6, pp. 65-76, 2001.

[20] T. Hufner, M. Kfuri, Jr., M. Galanski, L. Bastian, M. Loss, T. Pohlemann, and C. Krettek, "New indications for computer-assisted surgery: tumor resection in the pelvis," Clin. Orthop., pp. 219-225, 2004.

[21] T. Hufner, T. Pohlemann, S. Tarte, A. Gansslen, J. Geerling, N. Bazak, M. Citak, L. P. Nolte, and C. Krettek, "Computer-assisted fracture reduction of pelvic ring fractures: an in vitro study," Clin. Orthop., pp. 231-239, 2002.

[22] P. A. Grutzner and N. Suhm, "Computer aided long bone fracture treatment," Injury (Suppl. I), vol. 35, p. S-64, 2004.

[23] L. Joskowicz, C. Milgrom, A. Simkin, L. Tockus, and Z. Yaniv, "FRACAS: a system for computer-aided image-guided long bone fracture surgery," Comput. Aided Surg., vol. 3, pp. 271-288, 1998.

[24] "CJRR Report: Total Hip and Total Knee Replacements in Canada 2004," Canadian Institute for Health Information.

[25] "Norwegian Arthroplasty Register Annual Report 2004," Dep. Orthopaedics, Haukeland Univ. Hospital, Helse Bergen, Norway.

[26] G. van Hellemondt, M. de Kleuver, A. Kerckhaert, P. Anderson, F. Langlotz, L. Nolte, and P. Pavolv, "Computer-assisted pelvic surgery," Clin. Orthop., vol. 405, pp. 287-293, 2002.

[27] R. Bächler, H. Bunke, and L. P. Nolte, "Restricted surface matching-numerical optimization and technical evaluation," Comput. Aided Surg., vol. 6, pp. 143-152, 2001.

[28] P. Besl and N. McKay, "A method for registration of 3-D shapes," IEEE Trans. Pattern Anal. Mach. Intell., vol. 14, no. 2, pp. 239-256, Feb. 1992.

[29] B. Ma and R. E. Ellis, "Robust registration for computer-integrated orthopedic surgery: laboratory validation and clinical experience," Med. Image Anal., vol. 7, pp. 237-250, 2003.
[30] S. Lavallée, J. Troccaz, P. Sautot, B. Mazier, P. Cinquin, P. Merloz, and J.-P. Chirossel, "Computer-assisted spinal surgery using anatomy-based registration," in Computer Integrated Surgery: Technology and Clinical Applications, R. H. Taylor, S. Lavallée, G. C. Burdea, and R. Mösges, Eds. Cambridge, MA: MIT Press, 1996, pp. 425-446.

[31] S. Lavallée, "Registration for computer-integrated surgery: methodology, state of the art," in Computer-Integrated Surgery: Technology and Clinical Applications, R. Taylor, S. Lavallée, G. Burdea, and R. Mösges, Eds. Cambridge, MA: The MIT Press, 1996, pp. 77-97.

[32] N. Sugano, T. Sasama, Y. Sato, Y. Nakajima, T. Nishii, K. Yonenobu, S. Tamura, and T. Ochi, "Accuracy evaluation of surface-based registration methods in a computer navigation system for hip surgery performed through a posterolateral approach," Comput. Aided Surg., vol. 6, pp. 195-203, 2001.

[33] G. van Hellemondt, M. de Kleuver, A. Kerckhaert, P. Anderson, F. Langlotz, L. Nolte, and P. Pavolv, "Computer-assisted pelvic surgery. An in vitro study of two registration protocols," Clin. Orthop., vol. 405, pp. 287-293, 2002.

[34] S. Lavallée, P. Merloz, E. Stindel, P. Kilian, J. Troccaz, P. Cinquin, F. Langlotz, and L. P. Nolte, "Echomorphing: introducing an intraoperative imaging modality to reconstruct 3D-bone surfaces for minimally invasive surgery," in Proc. CAOS, 2004, pp. 38-39.

[35] E. Stindel, J. Briard, P. Merloz, S. Plaweski, F. Dubrana, C. Lefevre, and J. Troccaz, "Bone morphing: 3D-morphological data for total knee arthroplasty," Comput. Aided Surg., vol. 7, pp. 156-168, 2002.

[36] T. Hufner, J. Geerling, M. Kfuri, Jr., A. Gansslen, M. Citak, T. Kirchhoff, A. H. Sott, and C. Krettek, "Computer assisted pelvic surgery: registration based on a modified external fixator," Comput. Aided Surg, vol. 8, pp. 192-197, 2003.

[37] L. Ahrengart and U. Lindgren, "Heterotopic bone after hip arthroplasty. Defining the patient at risk," Clin. Orthop., vol. 293, pp. 153-159, 1993.

[38] B. Bierbaum, C. Hill, and J. Callaghan et al., "An analysis of blood management on patients having a total hip or knee arthroplasty," J Bone Joint Surg Am, vol. 81, pp. 2-10, 1999.

[39] T. Ault and M. Siegel, "Frameless patient registration using ultrasonic imaging," in Proc. First International Symposium on Medical Robotics and Computer-Assisted Surgery, 1994, pp. 74-82.

[40] C. Amstutz, M. Caversaccio, J. Kowal, R. Bachler, L. Nolte, and R. Hausler, "A-mode ultrasound-based registration in computer-aided surgery of the skull," Arch. Otolaryngol. Head Neck Surg., vol. 129, pp. 1310-1316, 2003.

[41] C. Maurer, R. Gaston, D. G. M. Hill, M. Taylor, M. Fenlon, P. Edwards, and D. Hawkes, "AcouStick: a tracked A-mode ultrasonography system for registration in image-guided surgery," in Proc. MICCAI, 1999, 1999, pp. 953-962.

[42] S. Schreiner, J. R. L. Galloway, J. Lewis, W. Bass, and D. Muratore, “An ultrasonic approach to localization of fiducial markers for interactive, image-guided neurosurgery. II. Implementation and automation," IEEE Trans Biomed Eng, vol. 45, pp. 631-641, 1998.

[43] B. Brendel, S. Winter, A. Rick, M. Stockheim, and H. Ermert, "Registration of 3D-CT and ultrasound datasets of the spine using bone structures," Comput. Aided Surg., vol. 7, pp. 146-155, 2002.

[44] G. Ionescu, S. Lavallée, and J. Demongeot, "Automated registration of ultrasound with CT images: application to computer-assisted prostate readiotherapy and orthopedics," in Proc. MICCAI 1999, 1999, pp. 768-777.

[45] J. Kowal, R. Hamdan, P. Heini, R. Kothe, L. Nolte, and M. Styer, "Ultrasound-based registration for minimially invasive interventions," in Proc. 3rd Annu. Meeting CAOS International, 2003, pp. 196-197.

[46] D. Muratore, J. Herring-Russ, B. Dawant, and R. Galloway, "Three-dimensional image registration of phantom vertebrae for image-guided surgery: a preliminary study," Comput. Aided Surg., vol. 7, pp. 342-252, 2002.

[47] D. Amin, T. Kanade, A. DiGioia, and B. Jaramaz, "Ultrasound registration of the bone surface for surgical navigation," Comput. Aided Surg., vol. 8, pp. 1-16, 2003.

[48] T. Hüfner, M. Oszwald, Jr., M. Kfuri, H. Rosenthal, M. Citak, and C. Kretter, "A-mode ultrasound registration in computer assisted pelvic surgery," in Proc. 3rd Annu. Meeting CAOS International, 2003, pp. $148-149$.

[49] J. Tonetti, L. Carrat, S. Blendea, P. Merloz, J. Troccaz, S. Lavallee, and J. P. Chirossel, "Clinical results of percutaneous pelvic surgery. Computer assisted surgery using ultrasound compared to standard fluoroscopy," Comput. Aided Surg, vol. 6, pp. 204-211, 2001. 
[50] J. Tonetti, L. Carrat, S. Blendea, J. Troccaz, J. Merloz, S. Lavallee, and J. P. Chirossel, "Clinical validation of computer assisted pelvic surgery using ultrasound. A percutaneous safe technique with low radiation exposure," Stud. Health Technol.Inform., vol. 81, pp. 515-520, 2001.

[51] B. Jaramaz, C. Nikou, B. Cavalier, S. Blendea, R. LaBarca, and A. M. DiGioia, "Experimental validation of ultrasound registration of long bones," in Proc. 3rd Annu. Meeting CAOS International, 2003, pp. $160-161$.

[52] G. Penney, P. Edwards, A. King, J. Blackall, P. Batchelor, and D. Hawkes, "A stochastic iterative closest point algorithm (stochastICP)," in Proc. MICCAI 2001, 2001, pp. 762-763.

[53] S. Heger, F. Portheine, J. Ohnsorge, E. Schkommodau, and K. Radermacher, "User-interactive registration of bone with a-mode ultrasound," IEEE Eng. Med. Biol. Mag., vol. 24, no. 2, pp. 85-95, Mar.-Apr. 2005.

[54] M. Siegel and T. Ault, "In situ calibration for quantitative ultrasonic imaging," IEEE Instrum. Meas. Mag., vol. 1, no. 3, pp. 9-18, Sep. 1998.

[55] F. Lindseth, T. Lango, J. Bang, and T. A. N. Hernes, "Accuracy evaluation of a 3D-ultrasound-based neuronavigation system," Comput. Aided Surg, vol. 7, pp. 197-222, 2002.

[56] M. Siegel, "Measurement issues in quantitative ultrasonic imaging," in Proc. IEEE Instrumentation and Measurement Technology Conf., 1997, pp. 16-19.

[57] J. Bamber, "Speed of sound," in Physical Principles of Ultrasonics, 2nd ed, C. Hill, J. Bamber, and G. ter Haar, Eds. Chichester, U.K.: Wiley, 2004, pp. 167-186.

[58] M. E. Anderson and G. E. Trahey, "The direct estimation of sound speed using pulse-echo ultrasound," J. Acoust. Soc. Am., vol. 104, pp. 3099-3106, 1998

[59] J. Krücker, J. Fowlkes, and P. Carson, "Sound speed estimation using automatic ultrasound image registration," IEEE Trans Ultrason Ferroelect Freq Contr., vol. 51, no. 9, pp. 1095-1106, Sep. 2004.

[60] D. Robinson, J. Ophir, L. Wilson, and C. Chen, "Pulse-echo ultrasound speed measurements: progress and prospects," Ultrasound Med. Biol., vol. 17, pp. 633-646, 1991.

[61] L. Mercier, T. Lango, F. Lindseth, and L. D. Collins, "A review of calibration techniques for freehand 3-D ultrasound systems," Ultrasound Med. Biol., vol. 31, pp. 143-165, 2005.
[62] R. Prager, R. Rohling, A. Gee, and L. Berman, "Rapid calibration for 3-D freehand ultrasound," Ultrasound Med. Biol., vol. 24, pp. 855-869, 1998.

[63] W. Thiel, "Ergänzung für die konservierung ganzer leichen nach W. Thiel," Ann. Anat., vol. 184, pp. 267-270, 2002.

[64] D. Barratt, A. Davies, A. Hughes, S. Thom, and K. Humphries, "Accuracy of an electromagnetic three-dimensional ultrasound system for carotid artery imaging," Ultrasound Med. Biol., vol. 27, pp. 1421-1425, 2001.

[65] A. Fitzgibbon, "Robust registration of 2D and 3D-point sets," in Proc. BMVC, 2001, pp. 411-420.

[66] U. D. Croce, A. Cappozzo, and D. Kerrigan, "Pelvis and lower limb anatomical calibration precision and its propagation to bone geometry and joint angles," Med. Biol. Eng. Comput., vol. 37, pp. 155-161, 1999.

[67] J. M. Fitzpatrick, J. West, and C. Maurer, "Predicting error in rigid-body point-based registration," IEEE Trans. Med. Imag., vol. 17, no. 5, pp. 694-702, Oct. 1998.

[68] J. M. Fitzpatrick, D. Hill, and C. Maurer, "Image registration," in Handbook of Medical Imaging, M. Sonka and J. M. Fitzpatrack, Eds, Washington: SPIE Press, 2000, vol. 2, Medical Image Processing and Analysis, p. 471.

[69] D. Hill and P. Batchelor, "Registration methodology: concepts and algorithms," in Medical Image Registration. ser. Biomedical Engineering, J. V. Hajnal, D. Hill, and D. Hawkes, Eds. London, U.K.: CRC Press, 2001, pp. 39-70.

[70] N. Pagoulatos, D. Haynor, and Y. Kim, "A fast calibration method for 3D-tracking of ultrasound images using a spatial localizer," Ultrasound Med. Biol., vol. 27, pp. 1219-1229, 2001.

[71] V. Daanen, J. Tonetti, and J. Troccaz, "Automatic delineation of the osseous interface in ultrasound by information fusion," in Proc. 7th Int Conf. Image Fusion, 2004, pp. 862-867.

[72] P. He and J. Zheng, "Segmentation of tibia bone in ultrasound images using active shape models," in Proc. 23rd Annu. Int. Conf. IEEE, 2001 pp. 2712-2715.

[73] A. Jain and R. Taylor, "Understanding bone responses in B-mode ultrasound images and automatic surface extraction using a Bayesian probabilistic framework," Proc. SPIE, pp. 131-142, 2004. 